Proceedings of the Edinburgh Mathematical Society (2002) 45, 693-700 (C)

DOI:10.1017/S0013091599001224 Printed in the United Kingdom

\title{
ON THE FREIHEITSSATZ IN CERTAIN ONE-RELATOR FREE PRODUCTS. III
}

\author{
ROBERT SHWARTZ \\ Department of Mathematics, Technion-Israel Institute of Technology, \\ Haifa 32000, Israel (robert@techunix.technion.ac.il)
}

(Received 19 October 1999)

\begin{abstract}
We study one-relator free products in which the relator has free-product length 4 . We find conditions for such presentations to have a Freiheitssatz and classify all non-aspherical presentations under certain conditions.
\end{abstract}

Keywords: Freiheitssatz; small cancellation; combinatorial group theory

AMS 2000 Mathematics subject classification: Primary 20F05; 20F06; 20F10

\section{Introduction}

Let $G_{1}$ and $G_{2}$ be groups. Let $r=a b c d \in G_{1} * G_{2}$, where $a, c \in G_{1}$, and $b, d \in G_{2}$. Let $G=G_{1} * G_{2} /\langle\langle r\rangle\rangle$, where we denote by $\langle\langle r\rangle\rangle$ the normal closure of $r$. We would like to classify the cases when the natural mappings $G_{1} \rightarrow G$ and $G_{2} \rightarrow G$ are embeddings. In other words, we would like to classify all the triples $\left(G_{1}, G_{2}, r\right)$ for which the Freiheitssatz holds (the classical case when $G_{1}$ and $G_{2}$ are both free being due to Magnus [13]).

The Freiheitssatz does not always hold, as the following example shows.

Example 1.1. $r=a^{2} b^{2} a^{-1} b^{-1},|a|=5$ and $|b|=7$, where we denote by $|x|$ the order of the element $x$ in the group $G$.

If $r=a^{2} b^{2} a^{-1} b^{-1}$, then we have $b a=a^{2} b^{2}$ in $G$, and then $b a^{2}=a^{2} b^{2} a=a^{2} b b a=$ $a^{2} b a^{2} b^{2}$. Since $b a^{2}=a^{2} b a^{2} b^{2}$, we get $b a^{2}=a^{2 m} b a^{2} b^{2 m}$, for every integer $m$. Since $|a|=5$, we get for $m=5, b a^{2}=a^{10} b a^{2} b^{10}=b a^{2} b^{10}$. Hence $b^{10}=b^{7}=1$ so $b=1$.

In [7] and [8], Howie showed that the Freiheitssatz holds true for the case $r=w^{n}$, where $n \geqslant 4$.

In [1], Duncan and Howie proved the Freiheitssatz for $r=w^{3}$, when $w$ does not contain a letter of order 2 .

In [9], Howie and Shwartz proved the Freiheitssatz for $r=\left(U a U^{-1} b\right)^{3}$, where $U$ is a word in $G_{1} * G_{2}$, and $a$ and $b$ are letters in $G_{1} \cup G_{2}$.

In [5], Edjvet and Juhasz have a classification of the cases where the Freiheitssatz holds if $r=a^{2} b^{2} a^{-1} b,|a|>10$ and $|b|>10$ or $r=a^{2} b^{2} a^{-1} b^{-1}$. 
In $[\mathbf{1 4}, \mathbf{1 6}]$, Shwartz has a classification for the cases of the Freiheitssatz for lengthfour one-relator free products, where the relator $r$ has the specific forms $r=a^{2} b a d$ and $r=a^{2} b a^{-1} d$.

This paper is the extension of the Freiheitssatz for length-four one-relator free products, where the relator has the generalized form $a b c d$.

We note here that one of the main applications of our work is for solving equations over groups. It turns out that the Freiheitssatz holds for $\left(G_{1}, G_{2}, r\right)$, where $r=a b c d$ if and only if the equation $a t b t^{-1} c t d t^{-1}$ has a solution over $G_{1} * G_{2}$. For more information on this subject the reader is referred to $[\mathbf{2}-\mathbf{4}, \mathbf{6}, \mathbf{1 0}, \mathbf{1 1}]$.

\section{Preliminaries}

We will make the following assumptions.

Let $G=G_{1} * G_{2} /\langle\langle r\rangle\rangle$, where $r=a b c d, a, c \in G_{1}$, and $b, d \in G_{2}$. Let $A$ be the subgroup of $G_{1}$ generated by $a$ and $c$, and let $B$ be the subgroup of $G_{2}$ generated by $b$ and $d$, and let $R_{A}$ be $\operatorname{Ker}(\langle a, c \mid-\rangle \rightarrow A)$, and let $R_{B}$ be $\operatorname{Ker}(\langle b, d \mid-\rangle \rightarrow B)$.

Assumption 2.1. $R_{A}$ contains none of $a^{e}, c^{e}, a^{f}, c^{f}, a c^{e}, a c^{f}, a^{2} c^{e}$, and $R_{B}$ contains none of $b^{e}, d^{e}, b^{f}, d^{f}, b d^{e}, b d^{f}, b^{2} d^{e}$, where $e \in\{-1,+1\}$ and $f \in\{-2,+2\}$.

The special cases when $R_{A}$ contains at least one of $a c^{f}$ or $a^{2} c^{e}$ or $R_{B}$ contains at least one of $b d^{f}$ or $b^{2} d^{e}$ have been studied in $[\mathbf{5}, \mathbf{1 4}, \mathbf{1 6}]$, and the other cases which appear in Assumption 2.1 are trivial.

Let $P=\left\{A_{4}, Z_{3} \oplus Z_{3}\right\}$ and let $R=\left\{A_{4}, S_{4}, A_{5}, Z_{3} \oplus Z_{3}, Z_{9}, Z_{12}, Z_{15}, Q_{12}\right\}$, where $A_{n}$ and $S_{n}$ denote the alternating and symmetric group of degree $n$ (respectively), $Z_{n}$ the cyclic group of order $n$ and $Q_{12}$ the quarternionic group of order 12, where $Q_{12}=$ $\left\langle a, c \mid a^{3} c^{2}, a^{6}, a c a c^{-1}\right\rangle$.

In this paper we shall prove the following theorems.

Theorem 2.2. Let $G=G_{1} * G_{2} /\langle\langle r\rangle\rangle$, and let $A$ and $B$ be the subgroups of $G_{1}$ and of $G_{2}$, respectively, as defined above, and suppose that Assumption 2.1 is satisfied. If $A \notin P$ and $B \notin R$, or if $A \notin R$ and $B \notin P$, then the Freiheitssatz holds for $\left(G_{1}, G_{2}, r\right)$.

Theorem 2.3. Let $G=G_{1} * G_{2} /\langle\langle r\rangle\rangle$, and let $A$ and $B$ be the subgroups of $G_{1}$ and of $G_{2}$, respectively, as defined above, and suppose that Assumption 2.1 is satisfied. If $A \notin R$ and $B \notin R$, and assume either $R_{1}=R_{A}$ and $R_{2}=R_{B}$ or $R_{1}=R_{B}$ and $R_{2}=R_{A}$, and $x y z w$ is a cyclic conjugate of abcd, where $\{x, y, z, w\}=\{a, b, c, d\}$, then $G$ is aspherical, unless one of the following holds.

(1) $x^{3}, z^{3} \in R_{1}$ and $y w y w \in R_{2}$.

(2) $x^{3}, z^{4} \in R_{1}$ and $y w y w \in R_{2}$.

(3) $x^{3}, z^{5} \in R_{1}$ and $y w y w \in R_{2}$.

In each of these cases there exists a non-trivial spherical van Kampen diagram. 


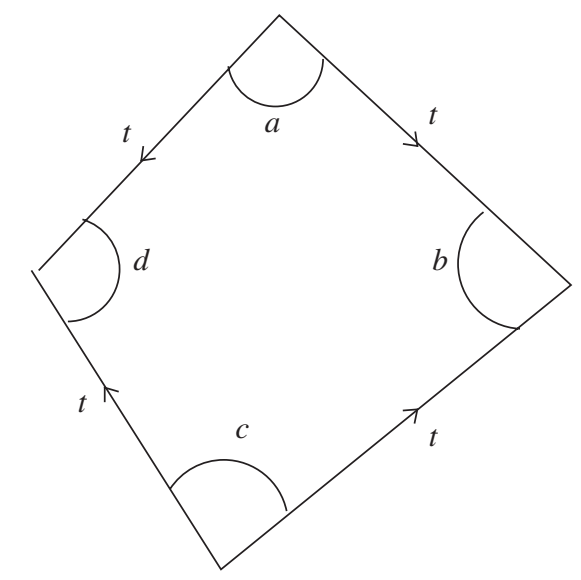

Figure 1. Labelling of a region in a modified relative diagram.

If Theorem 2.2 were false, then there would exist a van Kampen diagram (see Chapter 5 of [12] for undefined terms) over the presentation $A * B /\langle\langle r\rangle\rangle$ with boundary label $g$, where either $g \in A-\{1\}$ or $g \in B-\{1\}$. If Theorem 2.3 were false, then there would be a non-trivial spherical diagram over the presentation for $G$. Our method of proof in both cases is to show that no such diagrams exist.

Instead of working with van Kampen diagrams we will use a modified relative diagram which we now describe. Consider the presentation $\left\langle a, b, c, d, t \mid R_{A}, R_{B}, a t b t^{-1} c t d t^{-1}\right\rangle$. The diagram $M$, say, that we study will have regions given by Figure 1 (up to cyclic permutation and inversion).

Thus the edges are labelled by $t^{e}$, and the corners of the regions are labelled by $a^{e}$, $b^{e}, c^{e}, d^{e}$, where $e \in\{-1,+1\}$. Let $v_{0}$ be a distinguished vertex in the boundary of the diagram; the non-distinguished vertices will be called inner vertices, and an inner region is a region all of whose vertices are inner. The labelling is done in such a way as to ensure that the label $l(v)$, read anticlockwise around each inner vertex $v$, is a member of $R_{A}$ or $R_{B}$; and reading the labels clockwise around any region will give $\left(a t b t^{-1} c t d t^{-1}\right)^{e}$, where $e \in\{-1,+1\}$, up to cyclic permutation. If $D$ is a region of $M$, for $x \in\{a, b, c, d\}$ denote by $v_{x}(D)$ the vertex of $D$ whose corner is labelled $x$. If $v$ is a vertex of the region $D$, then $t_{D}(v)$ denotes the label of the corner at $v$ in $D$.

Thus if the Freiheitssatz fails for $G$, there will be a diagram $M$ as described above with distinguished vertex $v_{0}$ such that $l\left(v_{0}\right) \in A-\{1\}$ or $l\left(v_{0}\right) \in B-\{1\}$ (see [6]); and if Theorem 2.3 is false, then there exists a spherical $M$.

To prove that in each case no such diagram $M$ exists we use a curvature argument. If $S$ is a subdiagram of $M$ containing the vertex $v$, then $\|v\|_{s}$ denotes the valency of $v$ in $S$; if $S=M$ or if $S$ is clear from the context, we will use $\|v\|$. For each region $D$ of $M$ having $m$ vertices $v_{i}$, define the curvature $K(D)$ of $D$ by $K(D)=(1-m / 2)+\sum 1 /\left\|v_{i}\right\|$. Let $K(M)=\sum K(D)$. It is a consequence of the Gauss-Bonnet formula that $K(M)=2$. Our strategy will be to show that this cannot happen, thus obtaining our desired contradiction. 
Lemma 2.4. Suppose that $E$ is a region of $M$ containing the vertex $v$.

(a) If $t_{E}(v) \in\left\{a, a^{-1}, c, c^{-1}\right\}$, then $t_{D}(v) \in\left\{a, a^{-1}, c, c^{-1}\right\}$ for every region $D$ in $M$ that contains $v$.

(b) If $t_{E}(v) \in\left\{b, b^{-1}, d, d^{-1}\right\}$, then $t_{D}(v) \in\left\{b, b^{-1}, d, d^{-1}\right\}$ for every region $D$ in $M$ that contains $v$.

(c) If $\|v\|=3$ and $v \neq v_{0}$, then $l(v) \in\left\{a^{3}, b^{3}, c^{3}, d^{3}\right\}$.

(d) If $\|v\|=3$ and $v \neq v_{0}$, then $\|u\| \geqslant 4$ for every inner vertex $u$ which is adjacent to $v$.

\section{Proof.}

(a), (b) These follow from the fact that every vertex which is labelled by $a$ or $a^{-1}$ or $c$ or $c^{-1}$ is a source, and every vertex which is labelled by $b$ or $b^{-1}$ or $d$ or $d^{-1}$ is a sink. Hence every inner vertex is labelled by either a word in $R_{A}$ or a word in $R_{B}$.

(c) This is immediate from Assumption 2.1.

(d) By the symmetry between $a$ and $b$ and $c$ and $d$ in the rectangular region, we may assume without loss of generality that $l(v)=a^{3}$. Let $u$ be a vertex adjacent to $v$, and suppose that the edge connecting $u$ to $v$ is on the boundary of the regions $D_{1}$ and $D_{2}$. Then $t_{D_{1}}(v)=a$, thus $t_{D_{1}}(u)=b$ and $t_{D_{2}}(v)=a$, thus $t_{D_{2}}(u)=d$. Thus $b d$ is a subword of $l(u)$. Since $l(u)$ is mixed in $b$ and $d$, it follows that $\|u\| \geqslant 4$.

Remark 2.5. Since every inner region in the diagram is a rectangle, considering the Equation $(3.2)$ in $[\mathbf{1 2}$, p. 243] we have that $p=4$, and then by the equality $1 / p+1 / q=$ $1 / 2$, we get $q=4$ as well. Then the number of vertices in the boundary of the diagram is one. Hence we may assume that we are working in a spherical diagram, and every vertex in the diagram is an inner vertex.

Let $E$ be a region which satisfies $K(E) \geqslant K(D)$, for every $D \in M$.

For $x \in\{a, b, c, d\}$ denote by $v_{x}$ the vertex $v$ such that $t_{E}(v)=x$.

Without loss of generality we may make the following assumption.

\section{Assumption 2.6.}

(a) $\left\|v_{a}\right\| \leqslant\left\|v_{b}\right\|$.

(b) $\left\|v_{a}\right\| \leqslant\left\|v_{c}\right\|$.

(c) $\left\|v_{b}\right\| \leqslant\left\|v_{d}\right\|$.

\section{Lemma 2.7.}

(a) $\left\|v_{a}\right\|=3$.

(b) If $E$ is inner, then $l\left(v_{a}\right)=a^{3}$.

(c) If $E$ is inner, then $\left\|v_{b}\right\|=4$ or $5,4 \leqslant\left\|v_{d}\right\| \leqslant 11$. 
Proof. These observations follow almost immediately from our assumptions, the definition of the curvature, the fact that $K(E)>0$ and Lemma 2.4. For example, if $\left\|v_{d}\right\|>11$, then $K(E) \leqslant\left(1-\frac{4}{2}\right)+\left(\frac{1}{3}+\frac{1}{4}+\frac{1}{3}+\frac{1}{12}\right)=0$, a contradiction.

If $\operatorname{Conf}(E)=\left(\left\|v_{a}\right\|,\left\|v_{b}\right\|,\left\|v_{c}\right\|,\left\|v_{d}\right\|\right)$, and $E$ is again our region of maximal curvature, then it follows from Lemma 2.7 that the possibilities for $\operatorname{Conf}(E)$ are

(1) $(3,4,3,11)$ (in this case $\left.K(E)=\frac{1}{132}\right)$;

(2) $(3,5,3,7)$ (in this case $\left.K(E)=\frac{1}{105}\right)$;

(3) $(3,4,3,10)$ (in this case $K(E)=\frac{1}{60}$ );

(4) $(3,4,3,9)$ (in this case $\left.K(E)=\frac{1}{36}\right)$;

(5) $(3,5,3,6)$ (in this case $\left.K(E)=\frac{1}{30}\right)$;

(6) $(3,4,5,4)\left(\right.$ in this case $\left.K(E)=\frac{1}{30}\right)$;

(7) $(3,4,4,5)$ (in this case $\left.K(E)=\frac{1}{30}\right)$;

(8) $(3,4,3,8)$ (in this case $\left.K(E)=\frac{1}{24}\right)$;

(9) $(3,4,3,7)$ (in this case $\left.K(E)=\frac{5}{84}\right)$;

(10) $(3,5,3,5)$ (in this case $\left.K(E)=\frac{1}{15}\right)$;

(11) $(3,4,3,6)$ (in this case $\left.K(E)=\frac{1}{12}\right)$;

(12) $(3,4,4,4)$ (in this case $\left.K(E)=\frac{1}{12}\right)$;

(13) $(3,4,3,5)$ (in this case $\left.K(E)=\frac{7}{60}\right)$; and

(14) $(3,4,3,4)$ (in this case $\left.K(E)=\frac{1}{6}\right)$.

For $1 \leqslant i \leqslant 14$ the statement $\operatorname{Conf}(E)=i^{\prime}$ will mean that $\operatorname{Conf}(E)$ is case (i) in the above list.

\section{The idea of the proof}

The proofs of Theorems 2.2 and 2.3 are by case-by-case analysis of the 14 possible cases for $\operatorname{Conf}(E)$.

The idea of the proof is to look in every region $E$ which has positive curvature, and then to show that the average curvature of the diagram is less then 0 . We use the negatively curved neighbouring regions of $E$ to give compensation to $E$, and to other neighbouring positively curved regions. The proof involves repetitive calculations in each of the 14 cases. For this reason we give details of only one case below, and refer the reader to $[\mathbf{1 5}]$ for a complete account. 
The idea of the proof of one of the 14 cases is as follows.

Assume $E$ is a region which satisfies

(i) $\operatorname{Conf}(E)=(3,4,3,11)$; and

(ii) every region $D$ in the diagram satisfies $K(D) \leqslant K(E)$.

Lemma 3.1. Every region $D$, which satisfies $K(D)>0$, contains a vertex which has valency 11 .

Proof. Since every positively curved region $D$ should satisfy $K(D) \geqslant \frac{1}{132}$, and every $D$ satisfies $K(D) \leqslant K(E)=\frac{1}{132}$, we get $K(D)=\frac{1}{132}$. Then $\operatorname{Conf}(D)=(3,4,3,11)$. Then $D$ contains a vertex which has valency 11 .

Hence, if a region $D$ does not contain a vertex which has valency 11 , then $K(D) \leqslant 0$.

Let $E$ be any region which satisfies $K(E)=\frac{1}{132}$. Then $\operatorname{Conf}(E)=(3,4,3,11)$. Let $E_{i}$, where $1 \leqslant i \leqslant 10$, be the 10 regions other than $E$ that contain the vertex $v_{d}$. Let $v_{i}$ be the vertex adjacent to $v_{d}$ in $E_{i}$ for each $1 \leqslant i \leqslant 10$.

Lemma 3.2. If one of the $E_{j}$ has only one vertex which has valency 3 , then $\sum K\left(E_{i}\right)+$ $K(E) \leqslant 0$.

Proof. Let $E_{j}$ be the region which satisfies the condition that there is only one vertex in $E_{j}$ of valency 3 . Since every $E_{i}$ has a vertex $v_{d}$ which has valency 11 , then

$$
K\left(E_{j}\right) \leqslant \frac{1}{3}+\frac{1}{11}+\frac{1}{4}+\frac{1}{4}-1 \leqslant-\frac{10}{132} .
$$

Since every region $E_{i}$ satisfies $K\left(E_{i}\right) \leqslant \frac{1}{132}$, then

$$
K\left(E_{j}\right)+\sum K\left(E_{i}\right)+K(E) \leqslant-\frac{10}{132}+\frac{10}{132} \leqslant 0 .
$$

Lemma 3.3. $\sum K\left(E_{i}\right)+K(E) \leqslant 0$.

Proof. Assume $K(E)+\sum K\left(E_{i}\right)>0$. By Lemma 3.2, every $E_{i}$ contains at least two vertices of valency 3 . By Lemma $2.4(\mathrm{~d})$, no two adjacent vertices are of valency 3 . Thus if $K(E)+\sum K\left(E_{i}\right) \geqslant 0$, then every $E_{i}$ contains two opposite vertices of valency 3. Since $\left\|v_{d}\right\|=11,11$ vertices adjacent to $v_{d}$ each have valency 3 . Since every $v_{i}$ is adjacent to $v_{d}$, every $v_{i}$ is labelled by a word of length 3 in $a, a^{-1}, c$ and $c^{-1}$. Since $\left\|v_{i}\right\|=3$, then by Lemma 2.4 (a) $l\left(v_{i}\right) \in\left\{a^{3}, c^{3}\right\}$. Let $v_{0}$ be the vertex $v_{a}$. Thus $l\left(v_{0}\right)=a^{3}$. Now $v_{1}$ is opposite to $v_{0}$ in $E_{1}$, thus $l\left(v_{1}\right)=c^{3}$. Furthermore, $v_{i}$ is opposite to $v_{i-1}$ for every $i \leqslant 11$, thus if $l\left(v_{i-1}\right)=a^{3}$, then $l\left(v_{i}\right)=c^{3}$. Since $l\left(v_{0}\right)=a^{3}$, thus $l\left(v_{2 k}\right)=a^{3}$. Since $v_{10}=v_{c}$, we have $l\left(v_{10}\right)=c^{3}$, which is a contradiction to $l\left(v_{2 k}\right)=a^{3}$. Thus $\sum K\left(E_{i}\right)+K(E) \leqslant 0$. 
Now we turn to the proof of the case $\operatorname{Conf}(E)=(3,4,3,11)$. By Lemma 3.1, every positively curved region $D$ contains a vertex which has valency 11 . If a region $D$ contains a vertex which has valency 11, then according to the classification of the configurations, $\operatorname{Conf}(D)=(3,4,3,11)$. In particular, one vertex only in $D$ has valency 11 . Then it is enough to prove that if a region $D$ is positively curved, then the 11 neighbouring regions $D_{i}$ to $D$, which contain the vertex which has valency 11 in $D$, are giving enough compensation to $D$, considering that some of the neighbouring regions contain more then one vertex which has valency 11 , and then they should give compensation to regions other than $D$ as well. Assume a region $D_{1}$, which is a neighbouring region to $D$, has two or more vertices which have valency 11 . Then

$$
K\left(D_{1}\right) \leqslant \frac{1}{11}+\frac{1}{11}+\frac{1}{3}+\frac{1}{3}-1 \leqslant-\frac{5}{33} .
$$

Since every positively curved region has curvature $\frac{1}{132}, D_{1}$, by itself only, can give compensation to at least 20 regions, which is more than enough. Hence, we may assume that every neighbouring region $D_{i}$ to $D$ has one vertex only which has valency 11 . Then every $D_{i}$ needs to give compensation to $D$ only. Then by Lemma $3.3, \sum K\left(D_{i}\right)+K(D) \leqslant 0$, which proves the Freiheitssatz for the case where $\operatorname{Conf}(E)=(3,4,3,11)$.

Acknowledgements. I am grateful to Professor Jim Howie and to the referee for reading earlier versions of the paper and making useful remarks.

\section{References}

1. A. J. Duncan And J. Howie, Weinbaum's conjecture on unique subwords of nonperiodic words, Proc. Am. Math. Soc. 115 (1992), 947-954.

2. M. EDJvet, Solutions of certain sets of equations over groups, London Mathematical Society Lecture Note Series, vol. 159, pp. 105-123 (London Mathematical Society, 1991).

3. M. Edjvet, Equations over groups and a theorem of Higman, Proc. Lond. Math. Soc. 62 (1991), 563-589.

4. M. Edjvet And J. Howie, The solution of length four equations over groups, Trans. Am. Math. Soc. 326 (1991), 345-369.

5. M. Edjvet AND A. Juhasz, One-relator quotients of free products of cyclic groups, Commun. Alg. 28 (2000), 883-902.

6. J. Howie, The solutions of length 3 equations over groups, Proc. Edinb. Math. Soc. 26 (1983), 89-96.

7. J. Howie, The quotient of a free product of groups by a single high-powered relator, I, Pictures. Fifth and higher powers, Proc. Lond. Math. Soc. 59 (1989), 507-540.

8. J. HowIE, The quotient of a free product of groups by a single high-powered relator, II, Fourth powers, Proc. Lond. Math. Soc. 61 (1990), 33-62.

9. J. Howie AND R. Shwartz, One-relator products induced from generalized triangle groups, preprint.

10. F. Levin, Solutions of equations over groups, Bull. Am. Math. Soc. 68 (1962), 603-604.

11. R. C. Lyndon, On Dehn's algorithm, Math. Annln 166 (1966), 208-228.

12. R. C. LYNDON AND P. E. Schupp, Combinatorial group theory, Ergebnisse der Mathemtik und ihrer Grenzgebiete, vol. 89 (Springer, 1977).

13. W. Magnus, Über diskontinuierliche Gruppen mit einer definierenden Relation (Der Freiheitssatz), J. Reine Angew. Math. 163 (1930), 141-165. 
14. R. Shwartz, On the Freiheitssatz in certain one relator free products, I, Int. J. Alg. Computat. 11 (2001), 673-706.

15. R. Shwartz, On the Freiheitssatz in certain one relator free products, III, preprint (Heriot-Watt University Mathematical Reports Series HWM01-41 (2001), available at http://www.ma.hw.ac.uk/ jim/shwartz/HWM01-41.ps).

16. R. SHWARTz, On the Freiheitssatz in certain one relator free products, II, preprint. 